\title{
Leer, escribir y hablar como prácticas situadas. Un análisis de las literacidades de los jóvenes en el contexto escolar
}

\section{Reading, writing, and speaking as situated practices: an analysis of young people's literacies in the school environment}

DOI: https://doi.org/10.32870/dse.v0i24.1029

\section{Alma Karina Galindo Oceguera*}

Iparraguirre, M. S. y Malvestitti, M. (2018). Lectura, escritura y oralidad en la escuela. Prácticas comunicativas y de literacidad en estudiantes rionegrinos. Buenos Aires: UNRN.

La lectura, la escritura y la oralidad son prácticas situadas. Esto significa que leer, escribir y hablar son acciones que se realizan como parte de un marco contextual que abarca no sólo el escenario más inmediato, sino también el contexto histórico, social, económico y cultural en el que se encuentran inmersos aquellos que participan en esas prácticas (Barton, Hamilton, 2012). En el libro editado por María Iparraguirre y Marisa Malvestitti, la oralidad, la lectura y la escritura son contempladas desde un punto de vista multidimensional en el que, sin embargo, tiene un gran peso esta visión situada de la lengua, pues a lo largo de sus páginas se puede percibir una fuerte orientación hacia el entendimiento de la lengua como parte de fenómenos sociales que ocurren en el interior de las comunidades letradas.

La obra es el resultado de tres proyectos que abarcaron cinco años de trabajo realizado en varias localidades de la denominada Línea Sur de Río Negro, en Argentina, los cuales han sido fraccionados en siete documentos que integran los siete capítulos de la obra. De este modo, todos los capítulos presentados comparten no sólo el mismo marco teórico, sino también algunos de los métodos y los instrumentos para la construcción y análisis de los datos.

En el primer capítulo, Laura Eisner y María Iparraguirre plantean el marco teórico que encuadra los reportes presentados en los seis capítulos siguientes. El abordaje retoma, principalmente, elementos de la postura sociolingüística y de la perspectiva de los Nuevos Estudios de

* Doctora en Educación. Líneas de investigación: Literacidad y procesos educativos, Literacidad, lenguaje y cultura. México. almkary@yahoo.com.mx 
Literacidad (NEL). Por un lado, la postura sociolingüística enfatiza el vínculo que comparten los fenómenos sociales, lingüísticos y educativos; en ella se reconoce que las prácticas lingüísticas de los estudiantes y sus familias no siempre coinciden con las prácticas del ámbito escolar, lo que llega a ser una desventaja para aquellos que no participan del registro lingüístico hegemónico. Se retoma, también, la noción de "repertorio lingüístico-comunicativo", un concepto que nace de la sociolingüística y de la etnografía del lenguaje que alude al conjunto de estilos y variedades lingüísticas empleadas por una comunidad. Este repertorio lingüístico-comunicativo no solamente procede de los registros lingüísticos adquiridos en el interior de la comunidad a la que pertenecen los individuos, sino que también se alimenta de las interacciones que estos realizan en diferentes contextos, muchos de estos mediados, incluso, por las nuevas tecnologías.

Por otro lado, la corriente de los NEL, centrada en la exploración de las prácticas de lectura y escritura, sugiere que las prácticas de literacidad deben ser analizadas dentro del contexto sociocultural en el que se realizan. Desde esta perspectiva se señala, además, que hay múltiples literacidades que se corresponden con las diferentes áreas de la vida social, resultando así prácticas de literacidad dominantes en función de su aceptación, producción y reproducción en el interior de las instituciones hegemónicas. Estas prácticas de literacidad pueden ser estudiadas al analizar la serie de eventos de literacidad que las componen, es decir, los episodios sociales en los que la lectura, la escritura y la oralidad tienen un lugar preponderante, con el objetivo de extraer los sentidos y los usos que las personas designan a estas prácticas.

En general, los elementos que se acaban de reseñar funcionan como marco teórico para las investigaciones reportadas en los seis capítulos restantes, aunque algunos de estos trabajos se recargan más en una vertiente teórica que en otra. De cualquier modo, si se mira la obra de manera global, el lector se puede percatar de que, aunque cada una de las posturas con las cuales se atiende a un aspecto $u$ otro de la lectura, y la escritura o la oralidad tienen un peso distinto en cada capítulo, se logra mantener la cohesión teórica a lo largo de todo el libro.

En el segundo capítulo, Ana Atorresi, María Iparraguirre y Carlos Pardo exponen un estudio realizado en estudiantes preuniversitarios provenientes de entornos rurales y urbanos, que fueron sometidos a una prueba de lectura y a un cuestionario para el registro de datos culturales, económicos y sociales con la finalidad de identificar correlaciones entre los aspectos socioculturales de los jóvenes y sus resultados en la prueba de lectura. La mayor fortaleza de este trabajo es que los autores detallan minuciosamente las características de la prueba que elaboraron, en conjunto con los docentes de las escuelas participantes, con la finalidad de evaluar las habilidades de lectura de los estudiantes mediante ítems que abarcaron cinco niveles de dificultad y que iban desde la identificación literal de elementos en textos descriptivos breves, hasta la capacidad para realizar inferencias complejas o identificar la macroestructura global de textos de opinión y de divulgación científica. Los resultados mostraron que los jóvenes de entornos urbanos obtuvieron mayores puntuaciones en la prueba de lectura que los estudiantes de me- 
dios rurales; además, se observó que factores como un bajo nivel socioeconómico o un nivel de estudios inferior de los padres se vinculaba estrechamente con peores resultados en la prueba.

En el tercer capítulo, Laura Eisner reporta un estudio de caso que se enfoca, mediante una aproximación etnográfica, en las prácticas de escritura de jóvenes de educación media superior en un contexto escolar. El análisis realizado se centra en un evento de literacidad ocurrido en el contexto de una clase de Lengua y Literatura, a partir del cual la autora rescata los elementos que se ponen en juego durante el proceso de escritura, y que abarcan desde el uso que en este proceso se da a los artefactos de escritura como son los libros, hasta los roles asumidos y las negociaciones ocurridas en torno a qué y cómo se ha de escribir. La autora concluye que la puesta en juego de estos elementos durante la escritura tiene sus raíces en aspectos sociolingüísticos y socioculturales, lo que se puede traducir en que, al interactuar con diferentes elementos culturales y sociales propios de los medios en los que se desenvuelven, los jóvenes se dotan de todo un "repertorio de recursos" lingüístico-comunicativos que utilizan en sus decisiones y negociaciones en torno a la escritura.

En el cuarto capítulo Jimena Birgin, Gabriela Cabrera y Natalia Rodríguez exploran las prácticas de lectura de estudiantes de educación media a través de entrevistas y cuestionarios, e identificaron sus "cartografías lectoras", es decir, la organización de sus prácticas de lectura, los elementos que las componen y las interacciones entre ellos (Martos, 2010). Desde una mirada crítica hacia el concepto de juventud como un todo homogéneo, las autoras profundizan en los significados, valoraciones y representaciones que los estudiantes tienen hacia la lectura, identificando varios perfiles lectores que se caracterizaron por asociarla con el aprendizaje, la recreación, el ocio o el aburrimiento. Encontraron también que la práctica lectora es distinta en diferentes escenarios como la escuela y el hogar, en los que hay una implícita lucha entre lecturas oficiales y vernáculas que se manifiesta en el uso de los artefactos y soportes de lectura, su ubicación en los espacios de convivencia y la valoración que se le da a unas lecturas por sobre otras. Además, las autoras indagaron sobre el papel que la lectura tiene en la construcción de la subjetividad y la identidad, analizaron el rol que los padres y otros familiares pueden tener en la trayectoria lectora de los jóvenes e identificaron las características de las lecturas realizadas en escenarios virtuales como la red social Facebook.

Astrid Romero y Virginia Schuvab analizan, en el quinto capítulo, las decisiones que realizan los jóvenes cuando escriben textos argumentativos. Para ello, identificaron las particularidades de una serie de textos argumentativos escritos por estudiantes de educación media como parte de un ejercicio que les demandaba escribir una "nota de opinión". Entre ellas, se registró un uso predominante del borrador como estrategia de escritura, un extendido uso de la primera persona en los textos, junto con diversos recursos estilísticos y de argumentación empleados con la finalidad de convencer al lector sobre la postura planteada en el texto (p. ej. preguntas retóricas, negación, comparación, entre otros). De acuerdo con las autoras, el uso de estas he- 
rramientas para la escritura fue posible para los estudiantes debido a que éstas se encontraban "activas en sus repertorios" lingüístico-comunicativos, mismos que deberían ser rescatados por los currículos escolares para su máximo aprovechamiento en la enseñanza de las estrategias y habilidades para la argumentación.

En el sexto capítulo, Paula Adamo examina los grafitis realizados en las instalaciones de una escuela de educación media, con el objetivo de identificar los repertorios lingüístico-comunicativos de los jóvenes que se ponen en juego mediante esta práctica de escritura. La autora identificó cuatro categorías temáticas de los grafitis encontrados: aquellos vinculados con la violencia (groserías), la identidad (firmas), lo escolar (acordeones) y la territorialidad (declaraciones). Las pintas también se analizaron en función de las variaciones que éstas tuvieron con respecto al español estándar, como es el uso de palabras en el idioma inglés, acrónimos, grafías no convencionales y groserías. Resultan particularmente interesantes las evidencias ofrecidas que muestran el entrecruzamiento de los recursos propios de los contextos en internet y redes sociales (p. ej. el uso de emoticonos) y aquellos típicos de los grafitis, como el taggeo (firmas o etiquetas), de manera que se pueden reconocer pintas que contienen emoticonos al mismo tiempo que se puede taggear, es decir, firmar o etiquetar en línea. Ambas prácticas, además, se caracterizan por economizar recursos de manera que las palabras se abrevian, se omiten o se introducen grafemas que no corresponden con la escritura estándar, en un intento por emular las expresiones dadas en la oralidad.

En el último capítulo, Marisa Malvestitti y Mahe Ávila desmenuzan los recursos orales y escriturales de niños y jóvenes de poblaciones de los alrededores del lago Nahuei Huapi, una zona caracterizada históricamente por una amplia "interactividad lingüística" (es decir, una estrecha relación entre una o varias tradiciones lingüísticas que se influyen entre sí), centrándose en las variaciones lingüísticas producto de la convivencia entre hablantes del español y el mapuzungun, la lengua mapuche. Al analizar un corpus de producciones escritas y orales encontraron diferencias a nivel léxico ( $y$ otras, como la omisión de la "s" final en el uso del plural o la inconcordancia de género y número), entre el español de esa región y el español más apegado al canon hegemónico, considerando, hacia el final del capítulo, que estas variaciones sufren atenuaciones en estudiantes que están cerca de terminar sus estudios de educación media, lo que sugiere que el contacto con el español estándar en la escuela es un elemento a considerar en los procesos de socialización lingüística de los jóvenes.

Todos estos trabajos coinciden en que las variaciones de la lengua con respecto a los cánones hegemónicos durante las prácticas de lectura, escritura y oralidad de los jóvenes son aspectos que en el ámbito educativo deben ser revalorados y apreciados como producciones que tienen su origen en la cultura y el intercambio sociolingüístico característico de una región con particularidades y rasgos identitarios propios, pensamiento muy cercano al de los estudios decoloniales sobre literacidad, en los que se cuestiona el menosprecio hacia las prácticas de 
literacidad de los grupos sociales no hegemónicos (Hernández, 2019). Esta solidez ideológica de trasfondo da como resultado una obra redonda, en la que los autores no sólo comparten recursos y métodos sino que dialogan, de principio a fin, entre ellos y con los autores de su marco teórico, abriendo así una ventana para todos aquellos interesados en el estudio de las prácticas de literacidad, hacia los modos de leer, escribir y hablar de los jóvenes rionegrinos.

\section{Referencias}

Barton, D.; Hamilton, M. (2012). Local Literacies. Reading and Writing in One Community. Nueva York: Routledge.

Hernández, G. (2019). De los nuevos estudios de literacidad a las perspectivas decoloniales en la investigación sobre literacidad. Íkala, Revista de Lenguaje y Cultura, 24(2), 363-386. https:// revistas.udea.edu.co/index.php/ikala/article/view/334431/20793686

Martos, A. (2010). Las prácticas de lectura/escritura y los enfoques etnográfico y geográfico. Didáctica. Lengua y Literatura, 22, 199-229. https://revistas.ucm.es/index.php/DIDA/article/ view/DIDA1010110199A/18712 